\title{
The Yang-Mills vacuum wave functional in three dimensions at weak coupling
}

\section{Sebastian Krug* and Antonio Pineda}

Grup de Física Tè̀rica, Universitat Autònoma de Barcelona, E-08193 Bellaterra, Barcelona, Spain

E-mail: krug@ifae.es; AntonioMiguel.Pineda@uab.es

We compute the Yang-Mills vacuum wave functional in three dimensions at weak coupling with $\mathscr{O}\left(e^{2}\right)$ precision. We use two different methods to solve the Schroedinger functional equation. One of them generalizes to $\mathscr{O}\left(e^{2}\right)$ the method followed by Hatfield at $\mathscr{O}(e)$ [2]. The other uses the weak coupling version of the gauge invariant formulation of the Schroedinger equation and the ground state wave functional followed by Karabali, Nair, and Yelnikov [3]. We compare both results and discuss the differences between them.

Xth Quark Confinement and the Hadron Spectrum,

October 8-12, 2012

TUM Campus Garching, Munich, Germany

\footnotetext{
* Speaker.
} 


\section{Introduction}

In these proceedings we summarize the main results obtained in Ref. [1].

The determination of the ground-state (or vacuum) wave functional of QCD, $\Psi[A]$, is tantamount to solving QCD, as any observable (for instance the static potential or the spectrum of the theory) can then be obtained by the computation of the expectation value of the appropriate operator. Even if the exact solution is not known, properly chosen trial functions may give valuable information of the vacuum using variational methods (see for instance [4]).

We are still far from obtaining the exact ground-state wave functional of QCD. Even obtaining approximated expressions is very complicated. This is also true in the weak coupling limit. One reason follows from demanding the wave functional, besides of satisfying the Schroedinger equation, to be gauge invariant. This constraint is imposed by the Gauss law, and makes that standard quantum mechanics perturbation theory can not be applied. A procedure to overcome this problem was devised in the case of $\mathrm{SU}(2)$, and to $\mathscr{O}(e)$ in the weak coupling expansion, in Ref. [2]. An alternative procedure has also been considered in Ref. [5] and worked out to the same order in $e$.

Here we are interested in the three dimensional version of QCD without light fermions (i.e. Yang-Mills theory or gluodynamics). The method outlined by Hatfield [2] can also be applied to the three dimensional case and a general group $\mathrm{SU}(\mathrm{N})$ without major modifications. We do so and obtain the $\mathscr{O}(e)$ expression for a general group $\mathrm{SU}(\mathrm{N})$ in three dimensions. The result is in perfect agreement with the expression obtained by the expected replacements of the four dimensional result obtained in Ref. [2]. The results obtained with this method satisfy the Schroedinger equation by construction but not necessarily the Gauss law, though it can be explicitly shown that it does at $\mathscr{O}(e)$. We then compute the $\mathscr{O}\left(e^{2}\right)$ wave functional in what is a completely new result. Again, this result satisfies the Schroedinger equation by construction but at this order it's not possible to explicitly check the Gauss law, due to the complexity of the resulting expressions. The resulting wave functional is explicitly real (as expected for the ground-state functional) and we name it $\Psi_{G L}[\vec{A}]$.

The fact that gauge invariance can not be guaranteed in general is one important drawback of the previous method. The reason is that the Gauss law is only implemented partially for some terms in some intermediate expressions. Moreover, even this partial implementation of the Gauss law is difficult to automatize, as at each order it has to be tailored somewhat.

One solution to the previous problem would be to reformulate the Schroedinger equation in terms of gauge invariant variables. One such formulation was originally worked out in Refs. [6, 7 , 8, 9, 10] (for some introductory notes see [11]) and, more recently, in Ref. [3], where a modified approximation scheme was devised. The authors use a change of field variables, which become complex, to simplify the problem. Even though the original motivation of those works was to understand the strong coupling limit (the opposite limit we are considering here) and confinement in three dimensions, it is not difficult to see that the approximation scheme worked out in Ref. [3] could be easily reformulated to provide with a systematic expansion of the weak coupling limit. We do so and compute the ground-state wave functional to $\mathscr{O}\left(e^{2}\right)$. The vacuum wave functional is a function of the gauge invariant variables $J^{a}$, which we then transform to the original gauge variables $\vec{A}^{a}$. The resulting expression is gauge invariant by construction and also satisfies the Schroedinger equation by construction. We name it $\Psi_{G I}[\vec{A}] \equiv \Psi_{G I}[J(\vec{A})]$. Nevertheless, the explicit 
expression has the very unpleasant feature of having a non-trivial imaginary term.

We have then obtained two different expressions for the wave functionals: $\Psi_{G L}[\vec{A}]$ and $\Psi_{G I}[\vec{A}]$, which actually look completely different. At $\mathscr{O}(e)$ it is possible to show after several manipulations and using the symmetries of the integrals that both of them are equal (so at this order they are real and gauge invariant). Such brute force approach happens to be unfeasible at $\mathscr{O}\left(e^{2}\right)$ due to the complexity of the expressions. We need an organizing principle for the comparison. The approach we follow is to rewrite $\Psi_{G L}[\vec{A}]$ in terms of the gauge invariant variable $J^{a}$ and a gauge dependent field $\theta^{a}$. All $\theta^{a}$ dependent terms should vanish if $\Psi_{G L}[\vec{A}]$ is going to satisfy the Gauss law and we explicitly show that this happens. This means that both $\Psi_{G L}[\vec{A}]$ and $\Psi_{G I}[\vec{A}]$ are gauge invariant. We would then say that both should be equal, since both satisfy the Schroedinger equation. We actually find (after a rather lengthy computation) that both are almost but not completely equal. The difference is proportional to a bilinear real term. This is puzzling but there is a reason behind it: $\Psi_{G L}[\vec{A}]$ and $\Psi_{G I}[\vec{A}]$ satisfy "different" Schroedinger equations. $\Psi_{G L}[\vec{A}]$ was obtained using the unregulated Schroedinger equation, whereas $\Psi_{G I}[\vec{A}]$ was obtained after the Schroedinger equation in terms of $J^{a}$ variables was regularized. In this last case, regularization produces an extra term in the Schroedinger equation, producing in turn an extra term in the wave functional. Irrespectively of the above this comparison allows to rewrite $\Psi_{G I}[\vec{A}]$ in an explicitly real form. This is by far non-trivial, as the initial $\Psi_{G I}[J]$ was explicitly complex and dependent on complex variables. In particular there is a delicate cancellation between terms such that, after transforming this expression back to real variables, the wave function becomes real. This is an important test of several parts of the computation done in Ref. [3].

We believe that the weak coupling reformulation of the approach followed in Ref. [3] can be helpful to understand the meaning of the partial resummations performed in the approximation scheme used in this reference, though we do not explore this issue in this paper. Our $\mathscr{O}(e)$ or $\mathscr{O}\left(e^{2}\right)$ wave functional can also be used to test different trial functionals that one can find in the literature claiming to have the proper weak and strong coupling limit. Typically they reproduce the leading order weak coupling expansion but not the $\mathscr{O}(e)$ corrections. This is certainly the case with covariantization approaches where the exponent of the wave functional is approximated by a bilinear term in the $B$ fields (see for instance $[12,13]$ ). Therefore, our results can hint to how those trial functions could be improved to correctly incorporate corrections in the weak coupling limit.

\section{Results}

Because we are talking of the ground state we expect the wave function to be real and to have zero nodes. Therefore it can be written in the following way where F[A] does not diverge for finite A:

$$
\Psi[A]=e^{-F[A]}=e^{-F^{(0)}[A]-e F^{(1)}[A]-e^{2} F^{(2)}[A]+\mathscr{O}\left(e^{3}\right)} .
$$

\subsection{Determination of $\Psi_{G L}(\vec{A})$}

Working in the Hamiltonian formalism, we partially fix the gauge to $A_{0}=0$ and solve the 
functional Schroedinger equation in terms of the gauge fields ${ }^{1}$

$$
\frac{1}{2} \int_{x}\left(-\frac{\delta}{\delta \vec{A}^{a}(\vec{x})} \cdot \frac{\delta}{\delta \vec{A}^{a}(\vec{x})}+B^{a}(\vec{x}) B^{a}(\vec{x})\right) \Psi_{G L}[A]=E \Psi_{G L}[A],
$$

order by order in $e$, and at each order we implement the Gauss Law

$$
\left(\vec{\nabla} \cdot \frac{\delta}{\delta \vec{A}_{a}}+e f^{a b c} \vec{A}_{b} \cdot \frac{\delta}{\delta \vec{A}_{c}}\right) \Psi_{G L}[A]=0
$$

The details of this computation can be found in Ref. [1]. In summary we obtain the following expressions for the exponent of the wave functional:

$$
\begin{gathered}
F_{G L}^{(0)}[A]=\frac{1}{2} \int_{k} \frac{1}{E_{k}}\left(\vec{k} \times \vec{A}^{a}(\vec{k})\right)\left(\vec{k} \times \vec{A}^{a}(-\vec{k})\right), \\
F_{G L}^{(1)}[A]=i f^{a b c} \int_{k_{1}, k_{2}, k_{3}} \phi\left(\sum_{i=1}^{3} \vec{k}_{i}\right)\left\{\frac{1}{2\left(\sum_{i}^{3}\left|\vec{k}_{i}\right|\right)}\left(\vec{k}_{1} \times \vec{A}^{a}\left(\vec{k}_{1}\right)\right)\left(\vec{A}^{b}\left(\vec{k}_{2}\right) \times \vec{A}^{c}\left(\vec{k}_{3}\right)\right)\right. \\
\left.-\frac{1}{\left(\sum_{i}^{3}\left|\vec{k}_{i}\right|\right)\left|\vec{k}_{1}\right|\left|\vec{k}_{3}\right|}\left(\vec{k}_{1} \cdot \vec{A}^{a}\left(\vec{k}_{1}\right)\right)\left(\vec{k}_{3} \times \vec{A}^{b}\left(\vec{k}_{2}\right)\right)\left(\vec{k}_{3} \times \vec{A}^{c}\left(\vec{k}_{3}\right)\right)\right\},
\end{gathered}
$$

At $\mathscr{O}\left(e^{2}\right) F^{(2)}$ can have contributions from four and two fields (there are no contributions with three or one field, and the term with no fields can be absorbed in the normalization): $F^{(2)}[A]=$ $F^{(2,4)}[A]+F^{(2,2)}[A]$. The result we obtained reads

$$
\begin{aligned}
& F_{G L}^{(2,4)}=f^{a b c} f^{c d e} \int_{k_{1}, k_{2}, q q_{1}, q_{2}} \varnothing\left(\sum_{i}\left(\vec{k}_{i}+\vec{q}_{i}\right)\right) \frac{1}{\left|\vec{k}_{1}\right|+\left|\vec{k}_{2}\right|+\left|\vec{q}_{1}\right|+\left|\vec{q}_{2}\right|}\{ \\
& \frac{1}{2\left(\left|\vec{k}_{1}\right|+\left|\vec{k}_{2}\right|+\left|\vec{k}_{1}+\vec{k}_{2}\right|\right)\left(\left|\vec{q}_{1}\right|+\left|\vec{q}_{2}\right|+\left|\vec{q}_{1}+\vec{q}_{2}\right|\right)}\left\{\left(\vec{A}^{d}\left(\vec{q}_{1}\right) \times \vec{A}^{e}\left(\vec{q}_{2}\right)\right)\right. \\
& {\left[-\frac{1}{4}\left|\vec{k}_{1}+\vec{k}_{2}\right|^{2} \vec{A}^{a}\left(\vec{k}_{1}\right) \times \vec{A}^{b}\left(\vec{k}_{2}\right)+\frac{\left|\vec{k}_{1}+\vec{k}_{2}\right|}{\left|\vec{k}_{2}\right|}\left(\vec{k}_{1}+\vec{k}_{2}\right) \times \vec{A}^{a}\left(\vec{k}_{1}\right)\left(\vec{k}_{2} \cdot \vec{A}^{b}\left(\vec{k}_{2}\right)\right)\right.} \\
& \left.+\frac{\left(\vec{k}_{1}+\vec{k}_{2}\right) \cdot \vec{k}_{2}}{\left|\vec{k}_{1}\right|\left|\vec{k}_{2}\right|}\left(\vec{k}_{1} \cdot \vec{A}^{a}\left(\vec{k}_{1}\right)\right)\left(\vec{k}_{2} \times \vec{A}^{b}\left(\vec{k}_{2}\right)\right)+\left(\vec{k}_{1} \times \vec{A}^{a}\left(\vec{k}_{1}\right)\right)\left(\vec{k}_{1}+\vec{k}_{2}\right) \cdot \vec{A}^{b}\left(\vec{k}_{2}\right)\right] \\
& +\left(\vec{k}_{1} \times \vec{A}^{a}\left(\vec{k}_{1}\right)\right)\left(\vec{q}_{1} \times \vec{A}^{d}\left(\vec{q}_{1}\right)\right)\left(\vec{A}^{b}\left(\vec{k}_{2}\right) \cdot \vec{A}^{e}\left(\vec{q}_{2}\right)\right) \\
& +\frac{1}{\left|\vec{k}_{1}\right|\left|\vec{k}_{2}\right|}\left[2 \vec{k}_{2} \cdot \vec{A}^{e}\left(\vec{q}_{2}\right)-\frac{\vec{q}_{1} \cdot \vec{k}_{2}}{\left|\vec{q}_{1}\right|\left|\vec{k}_{2}\right|} \vec{q}_{2} \cdot \vec{A}^{e}\left(\vec{q}_{2}\right)\right]\left(\vec{k}_{1} \cdot \vec{A}^{a}\left(\vec{k}_{1}\right)\right)\left(\vec{k}_{2} \times \vec{A}^{b}\left(\vec{k}_{2}\right)\right)\left(\vec{q}_{1} \times \vec{A}^{d}\left(\vec{q}_{1}\right)\right) \\
& +\frac{1}{\left|\vec{k}_{1}\right|}\left(\vec{k}_{1} \cdot \vec{A}^{a}\left(\vec{k}_{1}\right)\right)\left(\vec{k}_{1}+\vec{k}_{2}\right) \times \vec{A}^{b}\left(\vec{k}_{2}\right)\left[\frac{1}{\left|\vec{q}_{2}\right|}\left(\vec{q}_{1}+\vec{q}_{2}\right) \times \vec{A}^{d}\left(\vec{q}_{1}\right)\left(\vec{q}_{2} \cdot \vec{A}^{e}\left(\vec{q}_{2}\right)\right)\right. \\
& \left.+\frac{2}{\left|\vec{q}_{1}+\vec{q}_{2}\right|}\left(\vec{q}_{1} \times \vec{A}^{d}\left(\vec{q}_{1}\right)\right)\left(\vec{q}_{1}+\vec{q}_{2}\right) \cdot \vec{A}^{e}\left(\vec{q}_{2}\right)\right]
\end{aligned}
$$

\footnotetext{
${ }^{1}$ In the following we use the notation $(d=2): \int_{x} \equiv \int d^{d} x, \int_{k} \equiv \int \frac{d^{2} k}{(2 \pi)^{d}}, \phi(\vec{k}) \equiv(2 \pi)^{d} \delta^{(d)}(\vec{k})$, and so on.
} 


$$
\begin{aligned}
& -\frac{2\left(\vec{q}_{1}+\vec{q}_{2}\right) \cdot \vec{q}_{1}}{\left|\vec{k}_{1}+\vec{k}_{2}\right|\left|\vec{k}_{1}\right|\left|\vec{q}_{1}\right|\left|\vec{q}_{2}\right|}\left(\vec{k}_{1} \cdot \vec{A}^{a}\left(\vec{k}_{1}\right)\right)\left(\vec{k}_{1}+\vec{k}_{2}\right) \times \vec{A}^{b}\left(\vec{k}_{2}\right)\left(\vec{q}_{1} \times \vec{A}^{d}\left(\vec{q}_{1}\right)\right)\left(\vec{q}_{2} \cdot \vec{A}^{e}\left(\vec{q}_{2}\right)\right) \\
& +\frac{2 \vec{k}_{1} \times \vec{k}_{2}}{\left|\vec{k}_{1}\right|\left|\vec{k}_{2}\right|\left|\vec{q}_{1}+\vec{q}_{2}\right|\left|\vec{q}_{2}\right|}\left(\vec{k}_{1} \cdot \vec{A}^{a}\left(\vec{k}_{1}\right)\right)\left(\vec{k}_{2} \times \vec{A}^{b}\left(\vec{k}_{2}\right)\right)\left(\vec{q}_{2} \times \vec{A}^{d}\left(\vec{q}_{1}\right)\right)\left(\vec{q}_{2} \times \vec{A}^{e}\left(\vec{q}_{2}\right)\right) \\
& +\frac{2}{\left|\vec{q}_{1}+\vec{q}_{2}\right|\left|\vec{q}_{2}\right|}\left(\vec{k}_{1} \times \vec{A}^{a}\left(\vec{k}_{1}\right)\right)\left(\vec{k}_{1}+\vec{k}_{2}\right) \times \vec{A}^{b}\left(\vec{k}_{2}\right)\left(\vec{q}_{2} \times \vec{A}^{d}\left(\vec{q}_{1}\right)\right)\left(\vec{q}_{2} \times \vec{A}^{e}\left(\vec{q}_{2}\right)\right) \\
& \left.+\frac{1}{\left|\vec{k}_{2}\right|\left|\vec{q}_{2}\right|}\left(\vec{k}_{2} \times \vec{A}^{a}\left(\vec{k}_{1}\right)\right)\left(\vec{k}_{2} \times \vec{A}^{b}\left(\vec{k}_{2}\right)\right)\left(\vec{q}_{2} \times \vec{A}^{d}\left(\vec{q}_{1}\right)\right)\left(\vec{q}_{2} \times \vec{A}^{e}\left(\vec{q}_{2}\right)\right)\right\} \\
& +\frac{1}{8}\left(\vec{A}^{a}\left(\vec{k}_{1}\right) \times \vec{A}^{b}\left(\vec{k}_{2}\right)\right)\left(\vec{A}^{d}\left(\vec{q}_{1}\right) \times \vec{A}^{e}\left(\vec{q}_{2}\right)\right) \\
& +\frac{1}{\left|\vec{k}_{1}\right|\left(\left|\vec{q}_{1}\right|+\left|\vec{q}_{2}\right|+\left|\vec{q}_{1}+\vec{q}_{2}\right|\right)}\left(\vec{k}_{1} \cdot \vec{A}^{a}\left(\vec{k}_{1}\right)\right)\left\{\frac{1}{2}\left(\vec{k}_{1}+\vec{k}_{2}\right) \times \vec{A}^{b}\left(\vec{k}_{2}\right)\left(\vec{A}^{d}\left(\vec{q}_{1}\right) \times \vec{A}^{e}\left(\vec{q}_{2}\right)\right)\right. \\
& -\left(\vec{q}_{1} \times \vec{A}^{d}\left(\vec{q}_{1}\right)\left(\vec{A}^{b}\left(\vec{k}_{2}\right) \times \vec{A}^{e}\left(\vec{q}_{2}\right)\right)\right. \\
& +\frac{1}{\left|\vec{q}_{1}+\vec{q}_{2}\right|\left|\vec{q}_{2}\right|}\left(\vec{k}_{1}+\vec{k}_{2}\right) \times \vec{A}^{b}\left(\vec{k}_{2}\right)\left(\vec{q}_{1}+\vec{q}_{2}\right) \times \vec{A}^{d}\left(\vec{q}_{1}\right)\left(\vec{q}_{2} \cdot \vec{A}^{e}\left(\vec{q}_{2}\right)\right) \\
& +\frac{1}{\left|\vec{q}_{1}\right|\left|\vec{q}_{2}\right|}\left(\vec{q}_{2} \times \vec{A}^{b}\left(\vec{k}_{2}\right)\right)\left(\vec{q}_{1} \cdot \vec{A}^{d}\left(\vec{q}_{1}\right)\right)\left(\vec{q}_{2} \times \vec{A}^{e}\left(\vec{q}_{2}\right)\right) \\
& \left.\left.+\frac{1}{\left|\vec{q}_{1}+\vec{q}_{2}\right|\left|\vec{q}_{2}\right|}\left(\vec{k}_{1}+\vec{k}_{2}\right) \cdot \vec{A}^{b}\left(\vec{k}_{2}\right)\left(\vec{q}_{2} \times \vec{A}^{d}\left(\vec{q}_{1}\right)\right)\left(\vec{q}_{2} \times \vec{A}^{e}\left(\vec{q}_{2}\right)\right)\right\}\right\}
\end{aligned}
$$

for the four-field term. For the two-field term a seemingly very complicated expression is obtained. Only after a careful comparison with the results of the next section is it possible to obtain the following compact equality

$$
F_{G L}^{(2,2)}=-N \frac{C_{A}}{4 \pi} \int_{k} \frac{1}{|\vec{k}|^{2}}\left(\vec{k} \times \vec{A}^{a}(\vec{k})\right)\left(\vec{k} \times \vec{A}^{a}(-\vec{k})\right),
$$

where

$$
N=0.025999(8 \pi)
$$

\subsection{Determination of $\Psi_{G I}(\vec{A})$ and comparison with $\Psi_{G L}(\vec{A})$}

The difficulties associated with the implementation of the Gauss law could be overcome by using a set of gauge invariant field variables and solving the Schroedinger equation in terms of these fields. The vacuum wave functional found in this way is explicitly gauge invariant. One such formulation was originally worked out in Refs. $[6,7,8,9,10]$ and the gauge invariant field used was

$$
J^{a}=2 i\left(M^{\dagger}\right)^{a c} A^{c}+\frac{2}{e}\left(\left(\partial M^{\dagger}\right) M^{\dagger-1}\right)^{a},
$$

where

$$
M^{\dagger}(x)=1+e \frac{4}{\vec{\nabla}^{2}}(\partial \bar{A})+e^{2} \frac{4}{\vec{\nabla}^{2}} \partial\left(\frac{4}{\vec{\nabla}^{2}} \partial \bar{A}\right) \bar{A}+\mathscr{O}\left(e^{3}\right)
$$

and

$$
A:=\frac{1}{2}\left(A_{1}+i A_{2}\right), \quad \bar{A}:=\frac{1}{2}\left(A_{1}-i A_{2}\right) .
$$


Out of this transformation the Hamiltonian obtained in these references was

$$
\begin{aligned}
H= & \frac{2}{\pi} \int_{w, z} \frac{1}{(z-w)^{2}} \frac{\delta}{\delta J_{a}(\vec{w})} \frac{\delta}{\delta J_{a}(\vec{z})}+\frac{1}{2} \int_{z}: \bar{\partial} J^{a}(z) \bar{\partial} J^{a}(z): \\
& +i e \int_{w, z} f_{a b c} \frac{J^{c}(w)}{\pi(z-w)} \frac{\delta}{\delta J_{a}(\vec{w})} \frac{\delta}{\delta J_{b}(\vec{z})}+\frac{e^{2} C_{A}}{2 \pi} \int J_{a}(\vec{z}) \frac{\delta}{\delta J_{a}(\vec{z})} .
\end{aligned}
$$

It is possible to obtain a recursive set of relations for the different components of the exponent of the vacuum functional [3]. We have checked those. An approximated solution was also given in Ref. [3] using a specific truncation scheme. We also checked this. Taking the weak coupling limit of this solution and transforming the vacuum wave functional back into the original gauge fields $\vec{A}^{a}$ allows us to compare it with the vacuum wave functional obtained in the previous section.

At $\mathscr{O}(e)$ and $\mathscr{O}\left(e^{2}\right)$ the resulting expressions are very large and in particular, they have nontrivial imaginary parts. They can be found in Ref [1]. Using the symmetries of the integrals one can show after some rewriting that the imaginary part of the $\mathscr{O}(e)$ term in this approach vanishes and that its real part is identical to Eq. (2.5). For the $\mathscr{O}\left(e^{2}\right)$ terms, however, this "direct" comparison is not feasible, as they are too large. We need an organizing principle that allows to split the comparison into pieces. The procedure we follow is to rewrite $\Psi_{G L}$ in terms of $J$ and $\bar{A}$ (actually we use a related variable named $\theta=\frac{1}{\bar{A}} \bar{A}+\ldots$ ). If $\Psi_{G L}$ and $\Psi_{G I}$ are going to be equal, all terms proportional to $\bar{A}$ (or $\theta$ ) should vanish. Moreover, to a given order in $e$ the polynomial in $\bar{A}$ is finite so only a finite number of terms need to be compared. This splitting simplifies the comparison considerably even though the calculations are still lengthy and tedious. Extra details can be found in Ref. [1].

In the end we find that $F^{(0)}, F^{(1)}$ and $F^{(2,4)}$ are real and agree with the corresponding expressions found above, while the bilinear term $F_{G I}^{(2,2)}[J(\vec{A})]$ is real but different from $F_{G L}^{(2,2)}[\vec{A}]$.

All together we obtain the following equalities

$$
F_{G L}[\vec{A}(J, \theta)]=F_{G I}[J]-\frac{C_{A} e^{2}}{4 \pi} \int_{k^{k}} \frac{\bar{k}^{2}}{|\vec{k}|^{2}} J^{a}(\vec{k}) J^{a}(-\vec{k})+\mathscr{O}\left(e^{3}\right),
$$

or in terms of the gauge fields

$$
F_{G I}[J(\vec{A})]=F_{G L}[\vec{A}]-\frac{C_{A} e^{2}}{4 \pi} \int_{\not k} \frac{1}{|\vec{k}|^{2}}\left(\vec{k} \times \vec{A}^{a}(\vec{k})\right)\left(\vec{k} \times \vec{A}^{a}(-\vec{k})\right)+\mathscr{O}\left(e^{3}\right) .
$$

The first equality implies that $F_{G L}[\vec{A}]$ is gauge invariant to $\mathscr{O}\left(e^{2}\right)$, the second that $F_{G I}[J]$ is real to $\mathscr{O}\left(e^{2}\right)$.

Overall we get complete agreement except for one bilinear real extra term in $F_{G I}$. Its origin can be traced back to the appearance of the last term of the Schroedinger equation in Eq. (2.12). In turn this term appears from an anomaly-like computation only after the kinetic operator has been regularized. Note that $F_{G L}$ was obtained without regularizing the theory, working with formal expressions. Therefore, even if the final result was finite, one could have missed contributions of this kind. For the other terms of $F$ we have got a double check, which gives us strong confidence in our result. 


\section{Conclusions}

We have computed the Yang-Mills vacuum wave functional in three dimensions at weak coupling with $\mathscr{O}\left(e^{2}\right)$ precision. We have used two different methods to solve the Schroedinger functional equation: (A) One of them generalizes to $\mathscr{O}\left(e^{2}\right)$ the method followed by Hatfield at $\mathscr{O}(e)$ [2]. We have named the result obtained $\Psi_{G L}[A]$. (B) The other uses the weak coupling version of the gauge invariant formulation of the Schroedinger equation and the ground-state wave functional followed by Karabali, Nair, and Yelnikov [3]. We have named the result obtained $\Psi_{G I}[A]$. Each method has its own strengths and weaknesses, and they are to some extent complementary.

The computations performed with method (A) are relatively simply and the results are explicitly real. The generalization to four dimensions of the $\mathscr{O}\left(e^{2}\right)$ computation does not present major conceptual problems. Note that this is the order at which we expect to start to see the running of the coupling constant in $D=4$. On the other hand, such a computation has two major drawbacks. First, the implementation of the Gauss law is not done in a systematic way but partially implemented in some intermediate steps. Therefore, we cannot guarantee a priori that the final result satisfies the Gauss law. As the results rapidly grow in size and complexity, a direct check turns out to be unfeasible. Actually we were only able to check the Gauss law with the help of method (B). The major drawback however is that the computation has been performed with an unregulated kinetic operator. Whereas all computations can formally be carried out obtaining a finite result, some terms may be missed in this way.

The computations with method (B) are somewhat more involved. Rather lengthy expressions appear when we rewrite the wave functional in terms of the gauge fields $\vec{A}$, which, moreover, look complex. Trying to prove by brute force that the result is real turns out to be impossible. Actually we only manage to prove it after a careful comparison with the result of method (A). Moreover, a possible generalization to four dimensions does not look trivial. On the other hand, method (B) is particularly appealing, as it directly works with gauge-invariant degrees of freedom. Therefore the Gauss law is automatically satisfied and it is not necessary to impose this constraint. It should be noted that with this method the Schroedinger equation can be solved recursively. Therefore, it could be possible to automatize it and obtain the wave functionals at higher order with a combination of algebraic/numeric programing. Finally and most important is that the operators in this computation have been regularized. This produced nontrivial contributions.

We have compared both results. It is very difficult to show that they are equal in a direct way. The strategy we follow helps a lot, yet it continues to be extremely complicated to show the equivalence of two given expressions. As we have already mentioned, this comparison has allowed us on the one hand to prove that $\Psi_{G L}$ was indeed gauge invariant and on the other hand that $\Psi_{G I}$ was real. Most interestingly the agreement between both results is almost complete except for one extra term that appears with the method (B). This term shows up from an anomaly-like computation once the theory is regularized. Such a contribution does not show up in method (A). Apparently, this is due to the fact that no regularization was used in this computation. This result is potentially very interesting because it is precisely this term that produces the mass gap and a linearly rising potential in the strong coupling limit in Ref. [10]. Therefore, it's important to understand how (and if) such a term can be generated in a regulated version of the Schroedinger formalism in terms of the gauge fields, as this contribution has not been checked with an independent method so far. 
However, as regularization in the Schroedinger formalism with gauge variables is, to a large extent, uncharted territory, this requires a dedicated study beyond the aim of this work. Yet, we plan to address this issue in the near future.

\section{Acknowledgments}

We acknowledge discussions with D. Karabali and V.P. Nair. This work was partially supported by the spanish grants FPA2010-16963 and FPA2011-25948, and by the catalan grant SGR200900894.

\section{References}

[1] S. Krug and A. Pineda, arXiv:1301.6922 [hep-th].

[2] B. F. Hatfield, Phys. Lett. B 147, 435 (1984).

[3] D. Karabali, V. P. Nair and A. Yelnikov, Nucl. Phys. B 824, 387 (2010) [arXiv:0906.0783 [hep-th]].

[4] A. Kovner and J. G. Milhano, In *Shifman, M. (ed.) et al.: From fields to strings, vol. 1*121-187 [hep-ph/0406165].

[5] H. S. Chan, Nucl. Phys. B 278, 721 (1986) [Erratum-ibid. B 287, 852 (1987)].

[6] D. Karabali and V. P. Nair, Nucl. Phys. B 464, 135 (1996) [arXiv:hep-th/9510157].

[7] D. Karabali and V. P. Nair, Phys. Lett. B 379, 141 (1996) [arXiv:hep-th/9602155].

[8] D. Karabali and V. P. Nair, Int. J. Mod. Phys. A 12, 1161 (1997) [hep-th/9610002].

[9] D. Karabali, C. j. Kim and V. P. Nair, Nucl. Phys. B 524, 661 (1998) [arXiv:hep-th/9705087].

[10] D. Karabali, C. j. Kim and V. P. Nair, Phys. Lett. B 434, 103 (1998) [arXiv:hep-th/9804132].

[11] H. Schulz, arXiv:hep-ph/0008239.

[12] J. Greensite and S. Olejnik, Phys. Rev. D 77, 065003 (2008) [arXiv:0707.2860 [hep-lat]].

[13] J. Greensite, H. Matevosyan, S. Olejnik, M. Quandt, H. Reinhardt and A. P. Szczepaniak, Phys. Rev. D 83, 114509 (2011) [arXiv:1102.3941 [hep-lat]]. 